\title{
Zur Geschichte der kinetischen Wärmetheorie mit biographischen Notizen zu August Karl Krönig
}

\author{
Von Grete Ronge
}

\section{Einführung}

Im Jahre 1856 erschien in Poggendorffs Annalen der Physik und Chemie eine Abhandlung des Berliner Physikers August KarL Krönig unter dem Titel «Grundzüge einer Theorie der Gase». ${ }^{1}$ In dieser Schrift werden die Wärmeerscheinungen, soweit sie an gasförmigen Körpern auftreten, zurückgeführt auf eine den Gasmolekülen anhaftende Bewegung. Das Thema lag zu damaliger Zeit in der Luft. Besonders' in England waren Ansätze in gleicher Richtung gemacht worden. Aber sie gewannen nicht die Verbreitung, und sie hatten nicht die anregende Kraft der Krönigschen Arbeit. Erst diese wirkte gewissermaßen auslösend auf die Entwicklung der kinetischen Gastheorie und darüber hinaus auf den Ausbau einer kinetischen Theorie der Materie.

Die Bezeichnung «kinetisch» darf hier jedoch nicht irreführen. Der Gesichtspunkt, der um die Mitte des vergangenen Jahrhunderts eine Veränderung in die Auffassung von der «Natur der Wärme» brachte, ist nicht darin zu suchen, daß man das Auftreten von Wärme zur Bewegung kleiner Partikel in Beziehung setzte. Von alters her waren derartige Vorstellungen lebendig gewesen. Schon die Atomisten der Antike hatten die Existenz leicht beweglicher "Feueratome» angenommen ${ }^{2}$, und ihre Lehre hatte, durch GASSENDI im 17.Jahrhundert zu neuem Leben erweckt ${ }^{3}$, in der «Wärmestoffhypothese » des 18. Jahrhunderts eine natürliche Fortsetzung erfahren. KRöNIG und die Wärmetheoretiker seiner Zeit gingen vielmehr von der Annahme aus, daß nicht ein besonderer Wärmestoff existiere, sondern die Atome der sicht- und greifbaren Materie selbst in ständiger Bewegung begriffen seien und auf solche Weise die Wärmeerscheinungen hervorrufen.

1 Pogg. Ann. XCIX (1856) 315-322.

2 Der Begründer der antiken Atomhypothese ist Leukipp. Er hat nach 450 v. Chr. in Abdera an der thrakischen Küste eine Philosophenschule gegründet. Sein Schüler DEмоKRIT von Abdera (etwa 460-370 v. Chr.) hat seine Lehre ausgebaut. Über die Feueratome s. etwa bei Aristoteles, Von der Seele I, 2.

${ }^{3}$ Pierre Gassendi (1592-1655), Minoritenpater, war ab 1646 Professor der Mathematik in Paris. Über seine Wärmelehre s. Opera I: De calore et frigore. 
Auch dieser Gedanke war damals nicht neu. Er hatte seine Vorläufer. Von den mechanischen Weltsystemen der Renaissancezeit - die Namen Bacon $^{4}$ und Descartes ${ }^{5}$ sind in diesem Zusammenhang vor allem zu nennen - soll hier abgesehen werden; denn ihre Beziehungen zur Physik des 19. Jahrhunderts treten nicht so unmittelbar zutage. Im Jahre 1738 aber trat Daniel Bernoulli ${ }^{6}$ mit einer rein mechanischen Auffassung der Wärme hervor, die in bezug auf Form und Inhalt in enger Verwandtschaft zur neuzeitlichen kinetischen Wärmetheorie steht. Sie ist im 10. Kapitel der Hydrodynamica ${ }^{7}$ enthalten. Da sie einen frühen Versuch darstellt, das Problem mit mathematischer Strenge zu behandeln, ist sie von grundsätzlicher Bedeutung.

\section{Bernoullis Theorie der elastischen Fluida ${ }^{8}$}

Bernoulli geht von einer Modellvorstellung aus. Als hervorstechende Eigenschaften der luftartigen Stoffe sind Ausdehnungsbestreben und Kompressibilität zu betrachten. Ein Bild, das zu Schlußfolgerungen über die

${ }^{4}$ Francis Bacon (1561-1626) war englischer Staatsmann und Philosoph. Seine Wärmetheorie ist in seinem gegen die scholastische Philosophie gerichteten, 1620 erschienenen Werke Novum Organum Scientiarum enthalten. BAcon lehnt Disputation und aristotelischen Syllogismus als Mittel der Naturerforschung ab und empfiehlt die Methode der Induktion. Er erprobt das «neue Werkzeug» am Beispiel des Wärmephänomens und kommt unter dessen Anwendung zu dem Schluß, daß Wärme eine Bewegung der kleinen Materieteilchen sei. l.c., Buch II, XI ff.

5 René Descartes (1596-1650) ordnet seine Anschauung über die Wärme dem Gesetz von der Erhaltung der Bewegung unter, das er als das beherrschende Prinzip des gesamten Naturgeschehens ansieht. Er erklärt die Wärme als eine zitternde Bewegung der Teilchen des Erdelementes. Principia Philosophiae, Teil IV, 28 ff.

${ }^{6}$ Daniel Bernoulli (1700-1782), ein Sproß der Basler Gelehrtenfamilie Bernoulli, trieb medizinische, naturwissenschaftliche und mathematische Studien. 1725 ging er als Professor der Mathematik nach Petersburg. 1733 kehrte er in seine Vaterstadt Basel zurück, wo er zunächst Anatomie und Botanik lehrte und 1750 auch die Professur für Physik übernahm.

7 Nach Bernouluis eigenen Angaben ist der Entwurf zu seinem Lehrbuch Hydrodynamica während der Petersburger Zeit, also vor 1733, entstanden. Daher dankt er in einem Vorwort der Petersburger Akademie und widmet das Werk dem Schutzherrn der Akademie, dem Fürsten Ernst Johann von Livland, Kurland und Semgallen. s. Hydrodynamica, Widmung und Praefatio.

${ }^{8}$ s. Hydrodynamica, Sect. X: De affectionibus atque motibus fluidorum elasticorum, praecipue autem aëris, S. 200-243. Die allgemeine Theorie ist vorwiegend in den Paragraphen 1-6 enthalten. 
Natur der elastischen Flüssigkeiten geeignet sein soll, hat vor allem in den eben genannten Merkmalen mit ihnen übereinzustimmen. Eine mathematische Überlegung, die sich an eine dieser Forderung genügende Abstraktion anknüpfen läßt, führt zu Gesetzmäßigkeiten, die in gleicher Form aus den experimentellen Erfahrungen mit luftförmigen Körpern erhalten werden.

Gegeben sei ein zylindrisches Gefäß, das oben durch einen beweglichen Stempel verschlossen wird ${ }^{9}$. In dem Zylinder soll sich eine große Anzahl kugelförmiger Teilchen befinden, die mit einer translatorischen Eigenbewegung ausgestattet sind, so daß sie regellos nach allen Richtungen hin- und herfliegen. Der Stempel kann durch Auflegen von Gewichten mehr oder minder beschwert werden. Infolge ihrer Bewegung beanspruchen die eingeschlossenen Korpuskeln einen weit größeren Raum, als ihrem Eigenvolumen entspricht, das, wie aus Späterem hervorgeht, im Verhältnis zum verfügbaren Flugraum als verschwindend klein anzunehmen ist ${ }^{10}$. Die Partikel teilen den Gefäßwänden ständig Stöße mit und so auch dem Deckel, der durch diese Impulse gestützt und bei vorgegebener Fluggeschwindigkeit je nach aufliegendem Gewicht in einer gewissen Höhe gehalten wird. Als solche Ansammlungen fliegender Teilchen sind nach Bernouldis Meinung die elastischen Fluida zu betrachten, als deren Repräsentant die atmosphärische Luft angesehen werden kann.

Aus den Experimenten mit Luft ist bekannt, daß bei konstant gehaltenem «Wärmegrad» Vergrößerung des Gewichtes eine Kompression hervorruft, Gewichtsverminderung aber eine Vermehrung des Volumens zur Folge hat. Eine mathematische Betrachtung, die von dem Modell ausgeht, dient der Begründung dieses Verhaltens. Die Fähigkeit der bewegten Korpuskeln, bei Kompression einem vergrößerten Stempelgewicht das Gleichgewicht zu halten, beruht auf zweifacher Ursache. Erstens ist die Anzahl der Teilchen im Verhältnis zum Raum jetzt größer geworden, und zweitens wiederholt jegliches Teilchen wegen der engeren Nachbarschaft den Stoß jetzt öfter. Bernoulli vergleicht einerseits die Anzahl der Teilchen miteinander, die bei verschiedenen Zylinderhöhen - Ruhezustand vorausgesetzt - dem beweglichen Deckel unmittelbar benachbart wären, also als Stoßkörper in

${ }^{9}$ l.c., Tab. VIII, Fig. 56. Als Vorbild der Zylinder mit beweglichem Stempel, die später in der Geschichte der Dampfmaschine eine so große Rolle spielen, können OTtov.GuERICKES Zylinderabbildungen in den Experimenta nova ut vocantur Magdeburgica de vacuo spatio, Amsterdam 1672, S. 109 und 111, angesehen werden.

10 Das geht aus den Berechnungen in $\S 4$ und $\S 5$ des 10. Kapitels der Hydrodynamica hervor. 
Betracht kommen. Andererseits stellt er in Rechnung, daß die Teilchen jetzt dichter beisammen sind, und er macht den Ansatz, daß der mittlere Abstand (distantia media) ${ }^{11}$ zwischen den Teilchenoberflächen in umgekehrtem Verhältnis zur Zahl der Zusammenstöße steht, wobei er den Durchmesser der Teilchen als vernachlässigbar klein annimmt. Durch Kombination beider Gesichtspunkte findet er, daß die zusammendrückenden Gewichte sich zu den beanspruchten Räumen umgekehrt proportional verhalten, eine Beziehung, die sich in analoger Form aus den Versuchen mit luftförmigen Körpern ergibt ${ }^{12}$. Allerdings ist der Einklang mit der Erfahrung bisher nur an verdünnter Luft geprüft, und BERNouluI betont die Notwendigkeit weiterer Experimente für den Fall starker Kompression, in welchem die Größe der Teilchendurchmesser Bedeutung gewinnt. Die Übereinstimmung mit dem experimentell gefundenen Boyle-Mariotteschen Gesetz mußte zu damaliger Zeit das Hauptziel einer Wärmetheorie bilden. Den Naturphilosophen der Renaissancezeit gegenüber genoß Bernoulli den Vorteil, daß er zu Anreiz und Bestätigung seiner Spekulationen von diesen gesicherten Versuchsergebnissen Gebrauch machen konnte, die den Früheren noch nicht zur Verfügung gestanden hatten.

Anschließend geht Bernoulli auf das Problem der Wärme ein ${ }^{13}$. Aus der Erfahrung ist bekannt, daß bei konstant gehaltenem Volumen die mit dem Barometer meßbare Elastizität, also der Druck der Luft, durch Wärmezufuhr zunimmt. Nach der obigen Modellvorstellung weist ein solches gemeinschaftliches Ansteigen darauf, daß eine Erhöhung des Wärmegrades mit einer Zunahme der Teilchengeschwindigkeit gleichbedeutend ist. Dabei ist offensichtlich, daß in eine mathematische Beziehung für den Druck die Geschwindigkeit $v$ mit dem Quadrat eingehen muß, weil durch Vermehrung der Geschwindigkeit sowohl die Anzahl der Stöße als auch deren Heftigkeit wächst. So wird das Verhalten der elastischen Flüssigkeiten auf die lebendige Kraft (vis viva) ihrer unwahrnehmbar kleinen Teilchen zurückgeführt. Der Druck ist der lebendigen Kraft proportional, und der Wärmegrad (gradus caloris), d.h. die Temperatur, ist der sinnfällige Ausdruck der mehr oder weniger lebhaften Korpuskelbewegung.

Die zunächst qualitative Beobachtung der Elastizitätssteigerung durch Erwärmung hatte der französische Physiker Amontons schon um 1700 zu

${ }^{11}$ s. l.c., $§ 4$.
12 s. l.c., 55.
13 s. l.c., 6. 
einer Gesetzmäßigkeit präzisiert ${ }^{14}$. Geht man von zwei Luftmengen aus, die zwar an Volumen gleich sind, sich aber in bezug auf die Dichten unterscheiden, so wächst, bei gleicher Steigerung des Wärmegrades in beiden Fällen, die Elastizität im Verhältnis der Dichten. Wenn auch Bernoullı die von АмоктоNs angegebenen Zahlenwerte für korrekturbedürftig hält, so erkennt er doch die Möglichkeit an, aus derartigen Messungen ein Maß der Wärme (mensura caloris) abzuleiten ${ }^{15}$.

Bernoullis gesamtes Werk der Hydrodynamica steht unter dem Leitgedanken, daß das Prinzip von der Erhaltung der lebendigen Kraft allgemeine Gültigkeit habe. Daher legt er an späterer Stelle des 10. Kapitels dar, in welcher Weise die an den elastischen Flüssigkeiten angestellten Beobachtungen im Sinne des Erhaltungsgesetzes zu verstehen seien ${ }^{16}$. Die Bedeutung, die das Thema für die Wärmemaschinentechnik besitzt, rechtfertige eine eingehende Behandlung. Den charakteristischen Unterschied zwischen dem luftförmigen Zustande und den anderen Erscheinungsformen der Materie sieht der Verfasser darin, daß die elastischen Flüssigkeiten vermöge ihrer Spannkraft andere schwere Körper zu heben imstande sind. Aus dieser Erfahrung leitet er die Berechtigung her, einer komprimierten elastischen Flüssigkeit - es ist dabei an ein gegebenes Quantum in gegebenem Zustande von Druck und Temperatur gedacht - eine bestimmte lebendige Kraft zuzuschreiben, und zwar soll mit der Bezeichnung «vis viva corpori elastico compresso insita $~{ }^{17}$ nichts anderes gesagt sein, als daß die elastische Flüssigkeit anderen Körpern einen Aufstieg zu vermitteln vermag, indem sie selbst Einbuße an elastischer Kraft erleidet. Der umgekehrte Vorgang der Kompression hat entsprechend einen Zuwachs des Fluidums an lebendiger Kraft im Gefolge.

14 Guillaume Amontons (1663-1705), Mitglied der Akademie der Wissenschaften zu Paris; s. Discours sur quelques proprietez de l'air et le moyen d'en connoître la temperature dans tous les climats de la terre, Histoire de l'Académie Royale des Sciences, Année 1702, avec les Memoires de Mathématique et de Physique pour la même Année, Amsterdam 1707, S. 204. ff.

15 Amontons' Bestimmung des absoluten Nullpunktes s. l. c. für das Jahr 1703, Amsterdam 1707, S. 61 ff. Амоnтons bestimmt den Grad der «äußersten Kälte», auf CelsiusSkala umgerechnet, zu $-239^{\circ} \mathrm{C}$. s. hierzu E. GerLand, Beiträge aus der Geschichte der Chemie, herausgegeben von Paul Diergart, Leipzig und Wien 1909, S. 359, 360. Bernoullis Bemerkungen zu Amontons' Messungen s. Hydrodynamica, Sect. X, $§ \S 7$ und 8.

${ }^{16}$ s. Hydrodynamica, Sect. X, § $39 \mathrm{ff}$.

17 s. l.c., S. 228. 
Gedankenexperimente mit dem Zylinder, in welchem der mit Gewichten beschwerte Stempel auf- und niedergeht, bilden den Ausgangspunkt quantitativer Überlegungen. Sie enthalten Ansätze zu den heutigen Betrachtungen der phänomenologischen Thermodynamik. Grundlegend für die Berechnungen ist LeIBNIzens Idee, daß die lebendige Kraft eines Körpers seiner Fallhöhe proportional ist. Bernoulli weist darauf hin, daß nicht absolute Werte von Druck und Wärmegrad für den Erfolg der Wärmemaschine ausschlaggebend sind, sondern daß eine Gleichgewichtsstörung erforderlich ist. Er hebt den hohen Betrag der in den Brennstoffen verborgenen lebendigen Kräfte hervor, die Fähigkeit des Heizmaterials, beim Verbrennen sowohl den Wärmegrad zu erhöhen als auch neue Mengen an elastischer Flüssigkeit zu erzeugen, und er macht auf die großen Kräfte aufmerksam, die bei der Verdampfung des Wassers entfesselt werden. Freilich waren zur Zeit, als die Hydrodynamica entstand, weder begriffliche Festsetzungen noch experimentelle Kenntnisse in der Wärmelehre genügend fortgeschritten, um hier exakte zahlenmäßige Angaben zu gestatten. Der Autor selbst ist sich der Problematik des gesamten Gebietes bewußt und bekennt, daß er seine Ansicht nur als physikalische Hypothese betrachte, über die noch nichts Bestimmtes ausgesagt werden könne. Er betont, daß das 10. Kapitel seiner Hydrodynamica neu sei, und er hofft, daß es der Erleuchtung und Lösung physikalischer Fragen dienen werde ${ }^{18}$.

\section{Die Wärmestoffhypothese}

Mit der kinetischen Theorie der Wärme war BeRnoulli seiner Zeit vorausgeeilt. Erst das 18. Jahrhundert brachte die eigentliche Entfaltung der experimentellen Wärmelehre, nachdem es durch die Erfindung und Entwicklung des Thermometers gelungen war, den durch die Temperatur charakterisierten Wärmezustand der Körper messend zu erfassen ${ }^{19}$. Erst

18 s. l.c., Sect. I, § 13.

19 s. hierzu BLACKs Vorlesungen über die Grundlehren der Chemie, deutsch von L.v. CReLL, Hamburg 1804, Band I, S. 67: «Unsere nächste Beschäftigung soll sein, die Natur des Thermometers zu erklären, ... das sowohl der Chemie als in der Arzneigelehrtheit sich außerordentlich nützlich bewiesen und uns in den Stand gesetzt hat, unsere Kenntnis von der Wärme ungemein zu erweitern und zu vervollkommnen.»An späterer Stelle faßt BLACK die drei Abschnitte «1) Erweiterung unserer Begriffe von der Wärme, 2) von der Verteilung der Wärme und 3) über die Schnelligkeit der Mitteilung der Wärme» unter der gemeinsamen Überschrift zusammen: «Fortschritte unserer Kenntnis von der Wärme vermittels des Thermometers.» l.c., S. $89 \mathrm{ff}$. 
jetzt bekamen grundlegende Begriffe wie Wärmegrad, Wärmemenge, Wärmekapazität eine präzise physikalische Bedeutung. Die experimentellen Erfahrungen aber, die unter Anwendung der neuen Hilfsmittel gesammelt wurden, schienen auf eine stoffliche Natur der Wärme zu deuten. Die Beobachtung, daß verschieden temperierte Körper bei Berührung Wärme austauschen, sprach für die Annahme eines fluiden Stoffes, der aus dem wärmeren in den kälteren Körper überströmt, bis Temperaturausgleich erfolgt ist.

$\mathrm{Da} ß$ aber auch die Wärmestoff hypothese im Grunde das makroskopische Geschehen auf Vorgänge im Bereich unwahrnehmbar kleiner Teilchen zurückführte, geht aus zeitgenössischen Darstellungen hervor. Die damals herrschenden Ansichten finden sich in BLAGKs Vorlesungen über die Grundlehren der Chemie ${ }^{20}$ erörtert, worin sich der Autor selbst für die Theorie ausspricht, daß sich die Wärmeerscheinungen aus dem Zusammenspiel zwischen den Korpuskeln der sicht- und greifbaren Materie und den Partikeln eines speziellen Wärmestoffes ergeben. Das wesentliche Unterscheidungsmerkmal zwischen den beiden Substanzarten liegt in den Kraftwirkungen, die sie untereinander ausüben. Während die Teilchen der Materie sich gegenseitig je nach Stoffart mit stärkerer oder geringerer Kraft anziehen, wohnen den Wärmestoffteilchen repulsive Kräfte inne, so daß sie sich gegenseitig abstoßen. Zwischen den Materieteilchen einerseits und den Wärmestoffteilchen andererseits sollen jedoch wiederum je nach Stoffart spezifische Anziehungskräfte wirksam sein ${ }^{21}$.

Die Wärmestoff hypothese verdankte ihre Erfolge vor allem ihrer großen Anwendungsfähigkeit und ihrer Anschaulichkeit. Die unterschiedliche

20 JoseF BLACK (1728-1799), Arzt und akademischer Lehrer in Glasgow und später in Edinburgh, hat seine Anschauungen über die Wärme und seine eigenen bedeutenden Entdeckungen auf diesem Gebiete nicht in Veröffentlichungen, sondern vorwiegend im Vorlesungsvortrag mitgeteilt. Sein Schüler, Dr.J.RoBison, gab die Vorlesungen unter Zuhilfenahme schriftlicher Hinterlassenschaften nach des Lehrers Tode heraus: Lectures on the Elements of Chemistry, 2 Bände, Edinburgh 1803. Die folgenden Zitatangaben beziehen sich auf die oben erwähnte deutsche Übersetzung von Dr.v. CReLl.

${ }^{21}$ l.c., Band I, S. 46/47. BLACK führt diese Theorie im Wesentlichen auf Dr. CleghorN zurück, einen jungen Gelehrten aus Edinburgh, der wenige Jahre nach seiner Promotion starb. Eine Diskussion ähnlicher Ansichten s. bei A.L. LAvorsier, Traité élèmentaire de chimie, Paris 1789, Tome I, S. 4, und bei John Dalton, New System of Chemical Philosophy, deutsche Übersetzung von FrIEdrICH WoLfF, Berlin 1812-1813, Band I, S. 3 und 160-163.

BLACK selbst warnt übrigens vor einer zu eingehenden Beschäftigung mit Modellvorstellungen. Er bezeichnet sie als müßige Grübeleien, die rein hypothetischen Charakter 
Wärmekapazität verschiedener Stoffe ließ sich durch die unterschiedliche Verwandtschaft zwischen den einzelnen Stoffarten und dem Wärmestoff erklären, die Wärmeausdehnung der Körper, ebenso das Ausdehnungsbestreben der Gase, durch die repulsiven Kräfte des aufgenommenen Wärmestoffes, der Wärmeumsatz bei Phasenumwandlungen durch Bindung bzw. Freigabe eines bestimmten Quantums an Wärmestoff ${ }^{22}$. Daß bei den Änderungen des Aggregatzustandes der hinläufige Prozeß den gleichen Betrag an «latenter Wärme» einsaugt, den der Rückweg wieder zur Verfügung stellt, festigte die Ansicht, daß hier ein Erhaltungsgesetz im Spiele sei, daß die Wärme unerschaffbar und unzerstörbar sei, Eigenschaften, die seit je als vornehmliche Kennzeichen der materiellen Substanzen gegolten hatten ${ }^{23}$.

Zudem fügte sich die Wärmestoffhypothese den Vorstellungen ein, die im Gefolge der Newtonschen Physik für das naturwissenschaftliche Denken bestimmend wurden. Gerade die Ausstattung der Stoffteilchen mit Korpuskularkräften gehörte zu den maßgeblichen neuen Gesichtspunkten dieser Epoche. Indem man den kleinen Körperbausteinen Attraktionskräfte zuschrieb, ordnete man den Bereich der Materieteilchen dem Prinzip der allgemeinen Gravitation unter. Einige Körpereigenschaften, z.B. Festigkeit und Kohäsion, ließen sich daraus ableiten. Mit den anziehenden Kräften allein aber waren viele Phänomene wie die Erscheinung des Lichtes, der Wärme und der Elektrizität und auch die Eigenschaften der Gase nicht hinreichend zu erklären ${ }^{24}$. Der Ausweg, die Existenz fluider Substanzen

haben und die chemischen Kenntnisse wenig vermehren. BLAcks Vorlesungen, Band I, S. 243.

Eine «dynamische» Theorie, welche die Materie als homogene Masse und die Erscheinungen der Elektrizität, des Lichtes und der Wärme als Kraftäußerungen an den ponderablen Körpern auffaßte, fand bei der Mehrzahl der Physiker wenig nachhaltige Anerkennung. s. hierzu G.Tн.FechNer in J.В.Вıот, Lehrbuch der Experimentalphysik, 2. Auflage der deutschen Bearbeitung, Leipzig 1828, Band I, S. 397, 398.

${ }^{22}$ s. etwa zu Wärmekapazitäten: BLACKS Vorlesungen, Band I, von der Verteilung der Wärme, S. 99-109, besonders S. 106, 107; A.L.LAvoisier, Traité élémentaire de chimie, Tome I, S. 19, 20; John Dalton, New System of Chemical Philosophy, deutsche Übersetzung, Band I, S. 57.

Zu Ausdehnungsbestreben der Gase: BLACK, l.c., S. 220, 221 und 252, 253; Lavoisier, l.c., S. 24, 25; DaLton, l.c., S. 164-169.

Zu Phasenumwandlungen: BLACK, l.c., S. 199 und 207 ff.; LAvoisier, l.c., S. 17.

${ }^{23}$ s. BLACKS Vorlesungen Band I, S. 161 und 242-248.

${ }^{24}$ Bemerkenswert im Hinblick auf moderne Anschauungen ist eine Hypothese, die der Jesuitenpater Roger JosePH Boscovich (geboren 1711 in Ragusa, gestorben 1787 in 
anzunehmen, die als Träger weitere Eigenschaften und Kräfte, z. B. solcher von repulsiver Art, auf das physikalische Geschehen Einfluß nehmen, wurde durch die experimentellen Erfahrungen der Zeit nahegelegt. Zwar ergab sich bei genauer Nachprüfung, daß diese Stoffe imponderabel sein mußten, da sie, indem sie in die sicht- und greifbare Materie einzogen, deren Gewicht nicht veränderten, und diese Beobachtung führte zu kritischen Einwänden und, wie sich BLACK in bezug auf den Wärmestoff äußert, zu Bedenken gegen dessen Stoffnatur selbst ${ }^{25}$. Doch waren die Proteste nicht unüberwindlich, und der Gedanke der Imponderabilien wurde zur Gewohnheit, ja man kann sagen, zum eigentlichen Kennzeichen der physikalischen Theorien des 18. und des beginnenden 19. Jahrhunderts ${ }^{26}$. Neben den elektrischen Flüssigkeiten und dem Lichtstoff hatte der Wärmestoff nichts Befremdendes, zumal sich die Wärmeübertragung durch Strahlung als Analogon zur Lichtausbreitung betrachten ließ, für welche zu damaliger Zeit die Newtonsche Emissionstheorie maßgeblich war ${ }^{27}$. Freilich herrschte über die Beziehungen der imponderablen Stoffe untereinander keine Klarheit. Sie wurden viel diskutiert, ohne daß eine einheitliche Ansicht sich durchgesetzt hätte ${ }^{28}$.

Schwerer wogen Zweifel, die sich an das Phänomen der Reibungswärme knüpften. Schon BAcoN hatte argumentiert, die Wärme sei nicht als selbständiger Stoff anzusprechen, da sie durch Reibung hervorgerufen werden

Mailand) in seinem Werk Philosophiae naturalis Theoria, reducta ad unicam legem virium in natura existentium, Wien 1759, darlegt. BoscovicH macht für Eigenschaften und Verhalten der Materie eine einzige, zwischen punktartigen, ausdehnungslosen Atomen wirkende Kraft verantwortlich, die sich nur im Bereich sehr geringer Entfernungen als Abstoßung, bei merklicher Entfernung aber als Anziehung betätigt.

${ }^{25}$ BLAcks Vorlesungen, Band I, S. 63-66.

${ }^{26}$ s. hierzu J.B. Вiот, Lehrbuch der Experimentalphysik, 2. Auflage der deutschen Bearbeitung von G.Th. Fechner, Leipzig 1828, Band I, 1. Kapitel: Allgemeine Betrachtungen über die Materialität, besonders S.23, 24, 25; s. auch FECHNERS eigene Ansicht, l.c., S. $404 \mathrm{ff}$.

${ }^{27}$ Als einer der ersten hat Carl Wilhelm Scheele (1742-1786) die Wärmestrahlung experimentell untersucht. s. Chemische Abhandlungen von der Luft und dem Feuer (1777), $\S 55 \mathrm{ff}$.

${ }^{28}$ s. hierzu A.L.LAvoisien, Traité élémentaire de chimie, Tome I, S. 6: «La lumière est-elle une modification du calorique, ou bien le calorique est-il une modification de la lumière ? C'est sur quoi il est impossible de prononcer dans l'état actuel de nons connaissances...» Wegen der unterschiedlichen Wirkungen aber werden in LAvoisıers Werk Licht und Wärme unterschieden. 
könne ${ }^{29}$. Die Anhänger der Wärmestoff hypothese halfen sich hier mit der Annahme, daß durch Reibung wie auch durch Erschütterung und $\mathrm{Zu}-$ sammendrückung Wärmestoff aus den betroffenen Körpern ausgetrieben werde ${ }^{30}$. Dieser Auffassung trat um die Jahrhundertwende Graf Rumford entgegen. Seine bekannten Versuche mit der Bohrmaschine hatten den Sinn, die Erzeugung der Wärme durch Bewegung nachzuweisen ${ }^{31} .1799$ widmete Humphrey Davy der gleichen Frage seine erste Experimentalarbeit. Durch eine sinnreiche Versuchsanordnung zeigte er, daß beim Gegeneinanderreiben von Eisstücken eine Erzeugung der Schmelzwärme durch die Reibung stattfindet ${ }^{32}$.

$\mathrm{Zu}$ den Angriffen experimentierender Physiker kommen theoretische Erwägungen, die sich auf grundlegende physikalische Prinzipien beziehen. In einer Gegenüberstellung möglicher Erklärungsweisen gehen LAvoisiER und LAPLACE zu Beginn ihrer ersten "Abhandlung über die Wärme » ${ }^{33}$ auf eine mechanische Wärmevorstellung ein. Es wird hervorgehoben, daß sie

29 Francis Bacon, Novum Organum, Buch II, § 18.

${ }^{30}$ s. hierzu auch BlAcks Ansichten über die Metallbearbeitung, Blacks Vorlesungen, Band I, S. 177, 178.

31 Benjamin Thompson (1753-1814) trat 1785 in die Dienste des Kurfürsten Karl Theodor von Pfalzbayern und wurde von diesem zum Grafen RumFord ernannt. Im Rahmen seiner militärischen Pflichten hatte er Gelegenheit, in der Werkstatt des Militärzeughauses in München das Kanonenbohren zu beobachten. Die großen Wärmemengen, die hierbei auftraten, festigten in ihm die Ansicht, daß Wärme Bewegung sei, und regten ihn zu eigenen Experimenten an. Er ließ in einem Zylinder einen stumpfen Bohrer sich um eine Achse drehen, so daß auf dem Boden des Zylinders starke Reibung auftrat. Die Maschine wurde von Pferdekraft getrieben. Sie war in einem mit Wasser gefüllten Kasten untergebracht. Das Wasser kam nach 2 $\frac{1}{2}$ Stunden zum Kochen. Hier wurde von einem isolierten Körper in schier unerschöpflicher Fülle Wärme hervorgebracht, was gegen eine stoffliche Natur der Wärme sprach.

Auch widerlegte RuMFonD durch direkte Messungen die irrige Ansicht, daß die beim Bohren entstehende Metallfeile eine kleinere Wärmekapazität habe als das kompakte Metall und daß der Differenzbetrag als Reibungswärme hervortrete. RuMFoRD, An Inquiry Concerning the Source of the Heat Which is Excited by Friction, Phil. Trans. LXXXVIII (1798) $80 \mathrm{ff}$.

32 Humphry Davy (1778-1829), der nachmalige Professor der Chemie an der Royal Institution. s. Essay on Heat, Light and the Combinations of Light, enthalten in Contributions to Physical and Medical Knowledge, collected by Th. Beddoes, Bristol 1799.

33 Antoine Laurent Lavoisier (1743-1794) und Pierre Simon Laplace (1749-1827), Mémoires de l'Académie für das Jahr 1780, S. 355 ff. Eine deutsche Übersetzung erschien in Ostwalds Klassiker der exakten Wissenschaften, Band 40. Hierauf beziehen sich die Zitatangaben. 
mit dem allgemeingültigen Gesetz von der Erhaltung der lebendigen Kraft in Einklang sei. Faßt man nämlich die Wärme als Molekularbewegung auf, so ist sie «die lebendige Kraft», heißt es dort, "welche resultiert aus den unmerklichen Bewegungen der Moleküle eines Körpers. Sie ist die Summe der Produkte der Masse jedes Moleküls in das Quadrat seiner Geschwindigkeit ». ${ }^{34}$ Mit Nachdruck heben die Verfasser die Berechtigung und den hypothetischen Charakter beider Anschauungen hervor ${ }^{35}$, diskutieren aber anschließend ihre Experimente mit dem Eiskalorimeter vom Standpunkt der Wärmestoff hypothese aus, und in LAvoisiens Traité élémentaire de Chimie (Paris 1789) zählt der Wärmestoff (calorique) zu den fünf «substances simples... qu'on peut regarder comme les élémens des corps ${ }^{36}$.

Blieb die Wärmestoff hypothese demnach stets eine umstrittene Theorie, so war um die Jahrhundertwende ihre Vorherrschaft dennoch nicht ernstlich in Gefahr, und sie festigte sich noch in den folgenden Jahrzehnten. Da es gelungen war, eine Fülle von Erscheinungen in der Gestalt exakter Gleichungen wiederzugeben, war ein Gefühl großer Sicherheit aufgekommen, und es lag der damaligen Zeit näher, das Erhaltungsgesetz, welches in den mathematischen Formulierungen zum Ausdruck kam, auf stoffliche Vorgänge zu beziehen als auf ein mechanisches Modell bewegter Materieteilchen, dessen mathematische Einkleidung schwierig zu handhaben war und dessen experimentelle Bestätigung noch ausstand oder bei dem Stande der Kenntnisse noch nicht die notwendige Überzeugungskraft hatte ${ }^{37}$. So trug bei einer Abwägung des Für und Wider die Stoffauffassung den Sieg davon.

\section{Die mechanische Wärmetheorie}

Erst die Verkündung des allgemeinen Energieprinzips in seiner quantitativen Form entzog der Wärmestoffhypothese den Boden. Die Reihe berühmt gewordener Experimentalarbeiten, in welchen J.P.Joule seit 1843 auf verschiedenen Wegen die Gleichwertigkeit von Arbeit und Wärme nachwies ${ }^{38}$, erschütterte schließlich das Vertrauen in die stoffliche Natur und

${ }^{34} l . c .$, S. 6.

${ }^{35}$ l.c., S. $6,7$.

${ }^{36} \mathrm{~s}$. Band I, S. 192.

${ }^{37}$ s. hierzu BLACKS Vorlesungen, Band I, S. 107, 108.

${ }^{38}$ J.P. Joule (1818-1889) beschreibt in seiner 1843 erschienenen Veröffentlichung «On the Calorific Effects of Magneto-Electricity and the Mechanical Value of Heat», Phil. Mag. XXIII (1843) 263 ff., 347 ff., 435 ff., seine Experimente über die Drehung eines 
überzeugte die wissenschaftliche Welt von der Wandelbarkeit der Wärme. Das ureigene Merkmal der materiellen Substanzen, die Unerschaffbarkeit und Unzerstörbarkeit, ließ sich in bezug auf die Wärme nicht länger behaupten. Rumfords Bohrversuche und Davys Arbeit über das Eisschmelzen fanden jetzt Verständnis und Beachtung.

Die Erkenntnis, daß die Wärme als eine Form der Energie aufzufassen sei, hatte das Bemühen um eine andere Anschauung über die «Natur der Wärme», die den jüngsten Erfahrungen besser angepaßt wäre, zur natürlichen Folge. So ist es zu erklären, daß die Arbeiten, die für die Entwicklung der modernen kinetischen Theorie richtungweisend wurden, in den Beginn der zweiten Hälfte des 19. Jahrhunderts fallen. Sie sind durch ihren mathematischen Charakter ausgezeichnet. Für die Vorgänge im atomaren Bereiche, mit denen man den Ereignissen im Wahrnehmbaren zu entsprechen suchte, strebte man die gleiche formelmäßige Art der Beschreibung an, die in den Darstellungen der phänomenologischen Wärmelehre angewandt wurde. Der Übergang zur quantitativen Betrachtungsweise ist nicht allein dadurch verursacht, daß man jetzt über die zahlenmäßige Beziehung zwischen Arbeit und Wärme verfügte. Die Erneuerung des Atomgedankens durch Dalton hatte den Vorstellungen über die Struktur der Materie eine neue Richtung gegeben. Auf das Gesetz von den konstanten und multiplen Proportionen gestützt, hatte Dalton behauptet, daß die letzten Partikel jeder chemisch homogenen Substanz alle von gleicher Gestalt und von gleichem Gewicht seien ${ }^{39}$. Diese mit präzisen physikalischen Eigenschaften ausgestatteten Materiebausteine fanden als Individuen genügender Realität und ausreichender Kennzeichnung nun Eingang in mathematische Abstraktionen.

Einen Einblick in die Situation der damaligen Zeit gewährt eine Abhandlung Joules unter dem Titel «Einige Bemerkungen über die Wärme und

elektrischen Leiters zwischen den Polen eines Magneten, und er weist nach, daß die hierbei erzeugte Wärme zu der aufgewandten mechanischen Arbeit in einem konstanten Verhältnis steht, dessen Zahlenwert er angibt. In den folgenden Jahren sichert er das Ergebnis durch andere Methoden, so durch Untersuchungen über Temperaturveränderungen durch Verdünnung und Verdichtung der Luft, Phil. Mag. XXVI (1845) $369 \mathrm{ff}$., und durch die Arbeiten über die Wärmeentwicklung bei Reibung von Flüssigkeiten, Phil. Mag. XXVII (1845) 205 ff.; Phil. Mag. XXXI (1847) 173; Phil. Trans. 1850, 61 ff.

39 John DaLton (1766-1844), New System of Chemical Philosophy, deutsche Übersetzung Band I, S. 162: «Jedes Teilchen Wasser ist gleich jedem anderen Teilchen Wasser; jedes Teilchen Wasserstoff ist gleich jedem anderen Teilchen Wasserstoff» usw. 
die Konstitution der elastischen Flüssigkeiten ${ }^{40}$ Der Verfasser weist zu Beginn auf seine grundlegenden Experimente zur Bestimmung des mechanischen Wärmeäquivalents hin, die keinen Zweifel lassen, «daß Wärme und mechanische Kraft in einander verwandelt werden » können, und er zieht aus dieser Äquivalenz den Schluß, «daß Wärme entweder die lebendige Kraft wägbarer Teilchen ist, oder ein Zustand der Anziehung oder AbstoBung, der imstande ist, lebendige Kraft zu erzeugen ». ${ }^{41}$

Unter dem neuen Aspekt sind die Temperaturveränderungen, welche die Gase bei Verdichtung oder Verdünnung erleiden, einer eingehenden Untersuchung unterzogen worden. Es gelang Joule, durch quantitative Messungen die eingewurzelte Behauptung der Wärmestoff hypothese zu widerlegen, daß die Wärmekapazität der Körper mit dem Volumen wachse ${ }^{42}$. Darüber hinaus hat ihm die Arbeitsfähigkeit der gespannten Gase eine Einsicht in

${ }^{40}$ Some Remarks on Heat and Constitution of Elastic Fluids, Mem. Lit. Phil. Soc. Manchester IX (1851) $107 \mathrm{ff}$., wieder abgedruckt in Phil. Mag. XIV (1857) $211 \mathrm{ff}$.

Joules Arbeiten über «Das mechanische Wärmeäquivalent» kamen als gesammelte Abhandlungen in deutscher Sprache, übersetzt von J. W.Spenges, heraus, Verlag Vieweg, Braunschweig 1872. Auf diese Übersetzung beziehen sich die Zitatangaben.

41 l.c., S. 121.

42 Das Phänomen der Abkühlung bei adiabatischer Ausdehnung von feuchter Luft machte sich schon GUERICKE gegenüber in Form von Tröpfchen- und Nebelbildung bemerkbar, als er Luft aus einem Kolben in einen zweiten evakuierten Kolben überströmen ließ. Er gibt als Grund an, die durch das Herabsteigen verminderte Luft könne nicht mehr so viel Feuchtigkeit enthalten wie im ursprünglichen Zustande. Eine Temperaturänderung fältt noch nicht auf. Experimenta nova, Lib. III, Cap. XI.

Die gleiche Erscheinung begegnet Denis Papin. Einen Brief an Christian Huygens vom 16. August 1691, in welchem er den Bau seines Tauchbootes beschreibt, schließt er mit der Mitteilung einer Beobachtung: «C'est qu'on laisse sortir l'air pressé de nostre machine on se trouve incontinent dans un brouillard: de mesme qu'en tirant l'air avec la machine du vuide on voit d'abord des nuées qui se forment dans le recipient.» In einem Antwortbrief vom 2. November 1691 versucht HuYgens die Erscheinung zu deuten, aber nicht in dem Sinne, daß die austretende Luft sich abkühlt. s. E. GERLAND, Leibnizens und Huygens' Briefwechsel mit Papin, Berlin 1881, S. 178 und 183.

Joule zählt den schottischen Arzt Dr. Cullen (1710-1790), Professor an den Universitäten Glasgow und Edinburgh und Lehrer von JosEF BLACK, zu den ersten Beobachtern des mit den Volumenänderungen der Gase verbundenen Temperatureffektes. BLACK erwähnt bereits als «wohlbekannte Tatsache», «daß ein Thermometer unter der Glocke einer Luftpumpe sinkt, wenn die Luft verdünnt wird, und steigt, wenn sie verdichtet wird.» BLAcKs Vorlesungen Band I, S. 247.

Die Erbauer der Dampfmaschine nutzen die Arbeitsfähigkeit der adiabatischen Entspannung aus. s. Brief von James Watt an Dr. Small vom 28. Mai 1769, abgedruckt in 
die Konstitution der elastischen Flüssigkeiten vermittelt; denn die Experimente «zeigen, daß die Wärme elastischer Flüssigkeiten die ihnen innewohnende mechanische Kraft ist; und da bekanntlich die Temperatur eines Gases die elastische Kraft desselben bestimmt, so folgt, daß die elastische Kraft oder der Druck die Wirkung der Bewegung der konstituierenden Teile des Gases sein $m u ß »{ }^{43}$

\section{Die kinetische Theorie von Joule}

Joule wendet sich nun der Frage einer geeigneten Modellvorstellung zu. Er geht zunächst auf einen Vorschlag ein, den DAVY in seinem 1812 erschienenen Lehrbuch Elements of Chemical Philosophy vorbringt und der, dem großen Rufe des Autors entsprechend, vielfach diskutiert wurde. Danach sind die Wärmeerscheinungen zurückzuführen auf Schwingungen und Rotationen von Materieteilchen, und zwar sind die Teilchen der festen Körper nur zu schwingender Bewegung fähig, die Teilchen der tropfbaren Flüssigkeiten und der gasförmigen Stoffe außerdem noch zu Rotationen um die eigene Achse. Den Gasteilchen kommt die größte Schnelligkeit zu. Die Temperatur soll von der Schwingungsgeschwindigkeit abhängen. Zunahme der Wärmekapazität bedeutet Vergrößerung des Raumes, in welchem sich die Bewegung vollzieht ${ }^{44}$.

J.P.Muirhead, Mechanical Inventions of James Watt, London 1856, Vol. I, S. 62, und Patent vom 12. März 1782, abgedruckt in MuirheAd, l.c., Vol. III, S. 60 ff.

DaLton bestimmt die Temperaturveränderung, die mit Kompression von Luft auf die Hälfte ihres Volumens verbunden ist. s. New System of Chemical Philosophy, deutsche Übersetzung Band I, S. 144.

$\mathrm{Zu}$ einer gesetzmäßigen Erfassung der Vorgänge kam es jedoch noch nicht. Noch 1824 sagt S. CARnot: «Le changement de température occasioné dans les gaz par le changement de volume peut être regardé comme l'un des faits les plus importans de la physique, à cause des nombreuses conséquences qu'il entraîne, et en même temps comme l'un des plus difficiles à éclaircir et à mesurer par des expériences décisives. Il semble présenter dans plusieurs circonstances des anomalies singulières.»s. Réflexions sur la puissance motrice du feu, Paris 1824, Neudruck 1953, S. 31.

Die anschauliche Erklärung der Wärmehypothese ging dahin, daß das geringere Gasvolumen auch die geringere Aufnahmefähigkeit für Wärme habe und daß bei Kompression die überschüssige Wärme herausgepreßt werde wie Wasser aus einem Schwamm. Man suchte also ähnlich wie im Falle der Reibungswärme die Zuflucht bei einer Änderung der Wärmekapazität. s. Blacks Vorlesungen Band I, S. 245 ff., und JoHN Dalton, Nero System of Chemical Philosophy, deutsche Übersetzung Band I, S. 9.

43 s. Das mechanische Wärmeäquivalent, S. 123.

${ }^{44}$ s. l.c., S. 123. 
Joule, der seine eigenen Betrachtungen auf den gasförmigen Zustand beschränkt, ist von DAvys Theorie tief beeindruckt, und unter ihrem Einfluß hat er in Zusätzen zu früheren Veröffentlichungen die Rotation als Grundbewegung der Gasteilchen in Betracht gezogen ${ }^{45}$. Obwohl diese These «dem Boyle-Mariotteschen Gesetze und anderen Erscheinungen, die man an elastischen Flüssigkeiten bemerkt, völlig entspricht», gibt er jedoch jetzt, der einfacheren Behandlungsart wegen, einem anderen Bilde den Vorzug, welches davon ausgeht, «daß die Gasteilchen beständig in allen Richtungen mit großer Geschwindigkeit durch den Raum fliegen und so durch den Anprall an eine denselben begrenzende Oberfläche einen Druck ausüben. ${ }^{46}$ Als Urheber der Idee wird Herapath ${ }^{47}$ genannt.

Die schlichte Namensnennung an dieser Stelle verrät nichts von dem erbitterten Kampf, den der englische Physiker John Herapath in den Jahren 1821/22 um eine mechanische Wärmeauffassung geführt hat ${ }^{48}$. Wie schon aus Joules Darstellung hervorgeht, nimmt auch Herapath, soweit er sich auf Gase bezieht, von dem Bilde geradlinig dahinfliegender Korpuskeln seinen Ausgang und leitet daraus das Boyle-Mariottesche Gesetz und eine Abhängigkeit zwischen Temperatur- und Druckzunahme her. Zu den physikalischen Phänomenen, die er mit Hilfe des kinetischen Modells erklärt, gehören die adiabatischen Volumenänderungen der Gase, die er in der Weise deutet, wie sie später JouLE vorgetragen hat, und die unterschiedliche Wärmeleitfähigkeit verschiedener Gasarten, die er auf die unterschiedliche, vom Teilchengewicht abhängige Fluggeschwindigkeit zurückführt. Da er aber wenig glückliche Definitionen einführt, mit eigenwilligen Festsetzungen und Reformvorschlägen hervortritt und im Bestreben, möglichst viele physikalische Erscheinungen seiner Theorie untertan zu machen, voreilige und noch nicht ausgereifte Vermutungen ausspricht, wirkt er mehr

45 s. l.c., S. 54, 74, 79 und 80.

Joule will die Wärmeerscheinungen «den großen Entdeckungen FARADAYs anpassen, ... daß jedes atomische Element mit derselben absoluten Elektrizitätsmenge verbunden ist ». l.c., S. 74. Joule nimmt an, daß die «Elektrizitätsatmosphären» um ihre respektiven Atome mit großer Geschwindigkeit kreisen und daß deren Drehgeschwindigkeit die Wärme ausmache. Er verweist später, l.c., S. 124, auf die vollständige mathematische Darstellung dieses Gedankens durch W.J.M.RANKInE, Edinburgh Trans. XX, mehrere große Abhandlungen unter dem Obertitel: On the Mechanical Action of Heat.

${ }^{46}$ s. Das mechanische Wärmeäquivalent, S. 124.

47 s. l.c., S. 124. John Herapath (1790-1868), Mathematiker und Physiker.

48 Herapaths Abhandlungen über die Wärmelehre nebst den Stellungnahmen anderer Physiker zu seiner Theorie finden sich in Annals of Philosophy, New Ser., Vol. I-IV. 
befremdend als gewinnend. Erkenntniskritische Einflechtungen erwecken eher das Mißtrauen der Experimentalphysiker, als daß sie zur Überzeugung beitragen. Angriffe und Gegenangriffe nehmen scharfe Formen an. Aber bei mancher berechtigten Kritik deutet die allgemeine Reserve, auf welche Herapaths Theorie in der wissenschaftlichen Welt stieß, doch an, wie gering vor der Entdeckung des Energieprinzips das Bedürfnis nach einer Erneuerung der Wärmeauffassung war. Wäre schon damals die Krise der Wärmestoffhypothese ausgebrochen gewesen, so hätte man wohl das Neuartige aufgegriffen, dem möglichen Lösungsweg Entgegenkommen gezeigt und aus der Fülle der Ideen das herausgelesen, was ja auch wenige Jahrzehnte später als annehmbar beurteilt wurde.

Von den Schwierigkeiten seines Vorläufers läßt Joule nichts verlauten. Er selbst beschränkt sich auf das Gebiet der elastischen Flüssigkeiten und hält sich an eingeführte Begriffe. In einer mathematischen Behandlung des Gasmodells gelangt er zu befriedigender Übereinstimmung mit der Erfahrung und zu überraschenden Ergebnissen ${ }^{49}$. In ein würfelförmiges Gefäß (1 Fuß Kantenlänge) sei bei normaler Temperatur $\left(60^{\circ}\right.$ Fahrenheit) ein Quantum Wasserstoff eingeschlossen, so bemessen, daß es gerade den Druck von einer Atmosphäre hervorruft. Die Durchschnittsgeschwindigkeit der Teilchen soll festgestellt werden. In einer Überschlagsrechnung darf angenommen werden, daß im Mittel je ein Drittel des gesamten Wasserstoffs zwischen je zwei gegenüberliegenden Würfelseiten hin- und herpendelt, ohne daß gegenseitige Störungen auftreten. Aus der bekannten Masse des eingeschlossenen Wasserstoffs läßt sich die Geschwindigkeit berechnen, die die Gasteilchen befähigt, den Druck einer Atmosphäre zu verursachen, wobei die Beziehung benutzt wird, daß der Druck proportional ist dem Quadrat der Teilchengeschwindigkeit. Für den vorgelegten Fall findet Joule den Geschwindigkeitswert von $6225 \mathrm{Fu}$ pro Sekunde.

Die Hälfte der Wasserstoffmenge, im gleichen Volumen untergebracht, würde bei gleicher Teilchengeschwindigkeit nur den halben Druck ausüben. Das Boyle-Mariottesche Gesetz ist also erklärt, wenn angenommen wird, daß die Geschwindigkeit durch die Temperatur festgelegt ist. Da Druck und Temperatur einander proportional ansteigen und nach der kinetischen Auffassung der Druck proportional ist der lebendigen Kraft der Gasteilchen, so folgt auch Proportionalität zwischen Temperatur und lebendiger Kraft. Die Geschwindigkeiten bei verschiedenen Temperaturen müssen

${ }^{49}$ s. Das mechanische Wärmeäquivalent, S. $124 \mathrm{ff}$. 
sich dann wie die Quadratwurzeln aus den betreffenden absoluten Temperaturen verhalten. Die «absolute Wärme» einer Gasmasse bei gegebener Temperatur ist ihr gesamter Betrag an lebendiger Kraft bei dieser Temperatur. Der erstaunlich hohe Wert, den Joule für die Teilchengeschwindigkeit errechnet hat, gewinnt durch einen Vergleich mit der spezifischen Wärme des Wasserstoffs an Glaubwürdigkeit. Das Geschwindigkeitsquadrat eines bewegten Körpers ist proportional derjenigen Höhe, zu der sich der Körper vermöge seiner lebendigen Kraft gegen die Schwere erheben würde, Leibnizens Gedanke. Da nach der kinetischen Theorie die Geschwindigkeitsquadrate den absoluten Temperaturen proportional sind, läßt sich für zwei benachbarte Temperaturen, etwa $60^{\circ}$ und $61^{\circ} \mathrm{F}$, die Differenz der Geschwindigkeiten ermitteln, und daraus eine diesem Betrag entsprechende Höhendifferenz. Sie beträgt im Falle des Wasserstoffs 1160 Fuß. Die mechanische Arbeit, die notwendig ist, um 1 Pfund um diese Höhe zu heben, muß, in Wärmeeinheiten umgerechnet, gleich der spezifischen Wärme des Wasserstoffs sein, wenn die hypothetischen Voraussetzungen der gesamten Überlegung von der Natur erfüllt werden. Das errechnete Resultat von 1,506 Wärmeeinheiten stimmt mit der gemessenen spezifischen Wärme des Wasserstoffs so weit überein, daß Joules kinetische Theorie in ihm eine wertvolle Stütze hat.

In der Ermittlung der Teilchengeschwindigkeiten treten keine Gesichtspunkte auf, die nicht hundert Jahre vorher auch schon übersehbar gewesen wären. Dennoch ist das Problem erst durch die Ergebnisse der dazwischenliegenden Entwicklung spruchreif und einer Prüfung zugänglich geworden. Erst durch die Untersuchungen von Gay-Lussac zu Beginn des 19. Jahrhunderts war in das Gesetz über die Wärmeausdehnung der Gase und damit in die Festlegung des absoluten Nullpunktes einige Sicherheit gekommen ${ }^{50}$. Erst Lavoisier und Laplace stellten durch die Erfindung des Eiskalorimeters ein Werkzeug zur Verfügung, die spezifischen Wärmen der Gase zu messen $^{51}$. Indessen hatten die Bemühungen um exakte Daten, die zu Joules Zeit noch anhielten, annähernd übereinstimmende Resultate geliefert ${ }^{52}$. So ordnete sich Joules Geschwindigkeitsergebnis einem Gefüge von

${ }^{50}$ s. Annales de Chimie, 43 (1802).

51 s. A.L. Lavoisier und P.S.Laplace, Mémoires sur la chaleur, Mémoires de l'Académie pour l'Année 1780, $355 \mathrm{ff}$., und Recueil des Mémoires de chimie de Lavoisier, Tome I, S. $121 \mathrm{ff}$. Deutsche Übersetzung in Ostwalds Klassiker der exakten Wissenschaften, Band 40. 52 s. Das mechanische Wärmeäquivalent, S. 126 f. 
Beziehungen und Zahlenwerten ein, das zu Bernoullis Zeit noch fehlte. Der wesentliche Vorteil gegenüber Herapath bestand in der Kenntnis der Äquivalenzbeziehung.

Joule hat seine Bemerkungen über die Konstitution der Gase an wenig zugänglicher Stelle publiziert. ${ }^{53}$ Vorwiegend aus diesem Grunde blieb sein an quantitativen Aussagen so reicher Beitrag wenig beachtet und wurde zumal im Auslande nicht bekannt. Erst 1857 erschien auf Bitten und Anraten von Rudolph Clausius ${ }^{54}$ ein Neudruck im Philosophischen Magazin ${ }^{55}$. Inzwischen war in Poggendorffs Annalen Krönigs Wärmehypothese herausgekommen.

\section{Krönigs Theorie der Gase .}

Als KRöNIG im Jahre 1856 seine Abhandlung über die Gase veröffentlicht, rechnet er die kinetische Theorie der Wärme bereits zu den geläufigen Diskussionsthemen der Physik. «Es fehlt aber durchaus», heißt es in der Einleitung, «an einer klaren Anschauung darüber, wie diese Bewegung eigentlich beschaffen ist.» ${ }^{56}$ Die nachfolgend entwickelte Hypothese soll diese Lücke ausfüllen. Auch KröNIg beschränkt sich auf Aussagen über den gasförmigen Zustand. Die Grundvorstellung ist von rein kinetischem Charakter und derjenigen von Bernoulli und Joule analog. Die Gase bestehen aus Atomen, «welche sich verhalten wie feste, vollkommen elastische, mit gewissen Geschwindigkeiten innerhalb eines leeren Raumes sich bewegende Kugeln ». ${ }^{57}$ Oszillationen um eine Gleichgewichtslage werden für den gasförmigen Zustand ausdrücklich verneint ${ }^{58}$, sondern das Gasteilchen «bewegt sich in gerader Linie und mit konstanter Geschwindigkeit so lange fort, bis es gegen ein anderes Gasatom oder gegen eine feste oder flüssige Wand stößt. Namentlich findet zwischen zwei Gasatomen, die sich nicht berühren, keine gegenseitige Abstoßung statt ». ${ }^{59}$ Zwar ist die Bahn

${ }^{53}$ Mem. Lit. Phil. Soc. Manchester, IX (1851) $107 \mathrm{ff}$.

${ }^{54}$ s. hierzu RudolPH Clausius in seiner Abhandlung «Über die Art der Bewegung, welche wir Wärme nennen", Pogg. Ann. C (1857) 354, Anmerkung 1, und ergänzend R. CLAUsIus, Abhandlungen über die mechanische Wärmetheorie, 2. Abteilung, Braunschweig 1867, S. 230 .

55 Phil Mag., XIV (1857) 211.

56 s. Pogg. Ann. XCIX (1856) 315.

${ }^{57}$ l.c., S. 316.

${ }^{58}$ KRöNIG spielt hier offenbar auf DAvYs Modell an.

${ }^{59}$ l.c., S. 316. 
des einzelnen Atoms unregelmäßig und entzieht sich der Berechnung. «Nach den Gesetzen der Wahrscheinlichkeitsrechnung wird man jedoch statt dieser vollkommenen Unregelmäßigkeit eine vollkommene Regelmäßigkeit annehmen dürfen. » ${ }^{60}$

Um zu formelmäßigen Aussagen zu gelangen, denkt auch KRöNIG an ein bestimmtes Gasquantum, das in einem parallelepipedischen Gefäß mit den Kantenlängen $x, y, z$ eingeschlossen ist. Auch er hält das Eigenvolumen der Gasteilchen im Verhältnis zum Kastenvolumen für vernachlässigbar - die Partikel sollen «ruhend nur einen sehr kleinen Teil vom Inhalte des Kastens einnehmen » $^{61}$ - und faßt den Gasdruck als Stöße der fliegenden Korpuskeln gegen die Gefäßwand auf. Die Analogie zu den Vorgängern bezieht sich jedoch vornehmlich auf den Gedankeninhalt. Was die Form anbetrifft, so ist KröNigs Behandlung des Problems in überlegener Weise geeignet, die Grundzüge der neuen Anschauung zu vermitteln. Indem der Verfasser auf die ganz bestimmte Anzahl diskreter Partikel eingeht, die eine bestimmte Gasmasse ausmachen - er bezeichnet sie mit $n$ - lenkt er die Aufmerksamkeit direkt auf das atomare Geschehen. Dadurch wird seine Darstellung unmittelbar anschaulich. Handelt es sich um einen einheitlichen Stoff, so ist anzunehmen, daß diese Teilchen alle von gleicher Masse $m$ sind. Geht man von der durchschnittlichen Geschwindigkeit $c$ aus, so kann die Bewegungsgröße der stoßenden Korpuskel mit der präzisen Bezeichnung $m c$ belegt werden. Die im Hinblick auf eine Rechenvereinfachung schon von Joule verwendete Abstraktion, daß je 1/3 der gesamten Gasmasse sich parallel zu je einer Kante des einschließenden Gefäßes bewegt, ergibt in KröNIGs Ausdrucksweise $n / 3$ Partikel pro Koordinatenrichtung. Als Produkt aus dieser Anzahl, aus der Bewegungsgröße $m c$ und aus der durch Geschwindigkeit und Gefäßdimensionen bestimmten Anzahl der Stöße, die das einzelne Teilchen in der Zeiteinheit auf die Gefäßwand ausübt, läßt sich nach Division durch die Fläche der Druck in der Form

$$
p \text { prop } \frac{n m c^{2}}{v}
$$

angeben. Da $n, m$ und $c$ bei konstanter Temperatur feste Werte haben, ist dies das Mariottesche Gesetz.

${ }^{60}$ l.c., S. 316.

${ }^{01}$ l.c., S. 316. 
Ersetzt man in diesem Ausdruck die lebendige Kraft $m c^{2}$ durch die Temperatur $t$, so ergibt $\operatorname{sich}$ bei $v=$ konst. die vom Gay-Lussacschen Gesetz her bekannte Proportionalität zwischen $p$ und $t$ in der Form

$$
p \operatorname{prop} \frac{n t}{v}
$$

Es besteht also eine aus der Erfahrung sich herleitende Berechtigung, in dem Produkt $m c^{2}$ oder der lebendigen Kraft eines Atoms nichts anderes als die vom absoluten Nullpunkt an gezählte Temperatur zu sehen.

Wird das Gesetz auf zwei verschiedene Gasarten, aber unter den besonderen Bedingungen $p_{1}=p_{2}, v_{1}=v_{2}$ und $t_{1}=t_{2}$ angewandt, so folgt, daß auch für die Anzahl der Teilchen $n_{1}=n_{2}$ gelten muß. Das heißt, «von verschiedenen Gasen sind bei gleichem Druck und gleicher Temperatur im gleichen Raum gleich viel Atome enthalten ${ }^{61}$ - eine Stütze des 1811 von Avogadro aufgestellten Gesetzes, das erst in der zweiten Hälfte des 19. Jahrhunderts nach Klärung der Begriffe Atom und Molekül zu gebührender Anerkennung gelangte. Aus dem Vorangegangenen folgt unmittelbar, daß die Masse $m$ eines Gasatoms dem spezifischen Gewicht des betreffenden Gases proportional ist.

Anschließend nimmt Krörig zu der Frage Stellung, ob das Gewicht einer gegebenen Gasmasse von der Temperatur abhängig sei. BERNoulLI hatte die Schwere unter den charakteristischen Eigenschaften der elastischen Flüssigkeiten aufgezählt und ausgesagt, daß das Fluidum den Boden des einschließenden Gefäßes immer in der gleichen Weise belaste, unabhängig davon, welche elastische Kraft ihm eigen sei ${ }^{62}$. Nach der kinetischen Theorie aber hat auf den ersten Blick auch der Gedanke Berechtigung, es müsse eine Temperatursteigerung, die ja gleichbedeutend mit Steigerung der lebendigen Kraft $m c^{2}$ ist, auch eine Vergrößerung des Druckes im Gefolge haben, den jedes einzelne Gasteilchen auf die Erde ausübt. Das aber würde einer Gewichtsvermehrung des Gasteilchens gleichkommen. In einer kurzen Rechnung zeigt KRöNIG, daß ein in vertikaler Richtung sich bewegendes Gasteilchen bei ungehindérter Flugbahn unabhängig vom Wärmezustand einen Druck gleich $m g / 2$ gegen die Erde ausübt, da zwar die Geschwindigkeit $c$ mit der Temperatur steigt, aber wegen der längeren Flugbahn die Anzahl der Stöße gegen die Erde im gleichen Verhältnis geringer wird.

${ }^{61}$ l.c., S. 318.

${ }^{62}$ Hydrodynamica, Sect. X, $§ 1$ und $\S 2$. 
Ist die Bewegung des Teilchens durch Zusammenstöße beschränkt, so daß das Atom quasi zwischen zwei horizontal übereinanderliegenden Wänden pendelt, so ergibt sich aus einer analogen Überlegung der Druckunterschied nach oben und nach unten zu $m g / 2$. Er ist also ebenfalls unabhängig von der Teilchengeschwindigkeit, d.h. von der Temperatur. Da dieser Drucküberschuß nach unten das Gewicht ausmacht, so ist offenbar, daß unabhängig von der Temperatur «ein geschlossenes Gefäß, in welchem $n$ Atome von der Masse $m$ enthalten sind, dadurch die Gewichtsvermehrung $n m g / 2$ » erleidet ${ }^{63}$. Hieraus folgert KRöNIG, «daß das Archimedische Gesetz für Gase gültig sein und daß ein spezifisch leichteres Gas innerhalb eines schwereren emporsteigen muß und umgekehrt ${ }^{64}$

Die gesamte Wärmemenge, die in einer Gasmasse von $n$ Teilchen enthalten ist, läßt sich nach KRöNIG in der Form $Q=n m c^{2}=n t$ darstellen. Sind also verschiedene Gasarten gleicher Temperatur gegeben, doch in solchen Quantitäten, daß sie in der Anzahl der Teilchen übereinstimmen, so enthalten sie auch gleiche Wärmequantitäten. Überdies drückt die der obigen Gleichung entsprechende Differentialgleichung aus, daß «alle Gase nach dem Volumen gemessen eine gleiche und konstante spezifische Wärme haben ». ${ }^{65}$ Unstimmigkeiten zwischen Theorie und Erfahrung führt KRöNIG darauf zurück, daß die Formeln Zeitbeanspruchungen unberücksichtigt lassen, welche sich aus den Zusammenstößen der Teilchen untereinander ergeben. Zum Abschluß erfahren die Temperaturveränderungen, die mit Ausdehnung und Kompression der Gase verbunden sind, die gleiche Deutung, die ihnen schon von Bernoulli, Herapath und Joule gegeben wurde ${ }^{66}$.

Krönigs Publikation folgt dem Erstdruck der entsprechenden Abhandlung Joules um mehrere Jahre nach. Eine Beeinflussung wäre also durchaus in Betracht zu ziehen. Ebenso könnte auf Grund der weitgehenden Analogien vermutet werden, daß er sowohl als die englischen beiden Autoren aus Bernoullis Theorie der elastischen Fluida geschöpft haben. Eine nähere Untersuchung aber deutet auf völlige Selbständigkeit. Ein Referat in der Zeitschrift Fortschritte der Physik, Band 13 (1859), geht auf die Originalitätsfrage ein. Der Referent, Dr. Jochmann aus Berlin, berichtet

${ }^{63}$ Pogg. Ann. XCIX (1856) 319 und 320.

${ }^{64}$ l.c., S. 320.

${ }^{65}$ l.c., S. 320.

${ }^{66}$ l.c., S. 321 und 322 . 
über Joules «Bemerkungen über die Wärme und die Konstitution der elastischen Flüssigkeiten», die im Zusammenhange mit den kürzlich erschienenen Publikationen von Krönig und Clausius besondere Bedeutung gewonnen habe. Er läßt die Bemerkung folgen, daß die Arbeiten von HerAPath und Joule die Verdienste der deutschen Physiker nicht einschränken, «welche die Hypothese selbständig von neuem aufgestellt und weiter entwickelt haben ». ${ }^{67}$ Da JochmanN und Krönig beide in Berlin ansässig und in der Redaktion der gleichen Zeitschrift tätig waren, ist zu vermuten, daß der Referent sich mit seiner Behauptung auf die unmittelbare Auskunft Krönigs stützen konnte ${ }^{68}$. Aber auch ein Zusammenhang mit BernoullI ist nicht wahrscheinlich, denn dessen Gastheorie war damals kaum bekannt. Erst 1859 erschien in Pogg. Ann. ${ }^{69}$ eine Übersetzung der ersten Paragraphen des 10. Kapitels der Hydrodynamica und machte das Publikum mit einer Theorie bekannt, die hundertzwanzig Jahre vordem entstanden war. Von nun an erschien Bernoullis Name unter den Autoren der kinetischen Theorie ${ }^{70}$.

Die Frage, ob die englischen Physiker von Bernoull abhängig sind, kann ebenfalls verneint werden. Herapath hat, um seine umfangreichen Arbeiten dem Publikum zugänglicher zu machen, in einer Übersichtstabelle Postulate und Gesetze, eigene und von anderen Autoren stammende Experimente und Schlußfolgerungen zusammengestellt, die er für seine Theorie als bedeutungsvoll ansah ${ }^{71}$. Dabei beruft er sich, wenn möglich, auf die Autorität berühmter Vorgänger und Zeitgenossen, deren Namen er in einer nebenlaufenden Spalte anführt. Er hätte kaum darauf verzichtet, an dieser Stelle neben Newton, Boyle, Mariotte, De Luc, Black, Gay-Lussac, Dalton, Davy und vielen anderen auch Bernoulli als Geistesverwandten zu nennen, wenn ihm damals die Theorie der elastischen Fluida aus der Hydrodynamica vertraut gewesen wäre. Auch lassen die ersten Veröffentlichungen, in denen Herapath auf die Genesis seiner Hypothese eingeht, vermuten, daß der Verfasser seine Abhandlungen im Bewußtsein darbietet, der wissenschaftlichen Welt etwas ganz Neuartiges vorzutragen.

\footnotetext{
67 s. Fortschritte der Physik XIII (1859) 283.

${ }^{68}$ s. unten, Biographische Notizen zu August KarL Krönig.

${ }^{69}$ s. Pogg. Ann. CVII (1859) 490-494.

${ }^{70} \mathrm{~s}$. auch Rudolph Clausius, Abhandlungen über die mechanische Wärmetheorie, 2. Abteilung (1867), S. 230 und 231. CLAUSIUS weist hier in einem historischen Überblick vor allem auf den Genfer Mathematiker und Physiker Le SAGE (1724-1803) hin.

${ }^{71}$ s. Annals of Philosophy III (1822) $16 \mathrm{ff}$.
} 
Von der besonderen Eignung der KRöNIgschen Arbeit, die Physiker mit der kinetischen Wärmeauffassung bekannt zu machen, ist schon die Rede gewesen. Daher verlor sie auch nicht an Bedeutung, nachdem Joules Bemerkungen über die Konstitution der Gase durch neuerliche Publikation bekannt geworden waren. KröNIGs Gastheorie umfaßte nur sieben Druckseiten. In dieser Kürze wie in der Prägnanz der Darstellung mag der Erfolg mitbegründet gewesen sein. Da der Verfasser auf spezielle Beispiele verzichtete und seine mathematischen Ableitungen in allgemeinen Zahlen hielt, machte er die Theorie dem Verständnis leicht zugänglich und ließ den Anspruch auf Allgemeingültigkeit unmittelbar zum Ausdruck kommen. HeLmholtz schreibt darüber in einem Referat, «daß sie (KröNigs Hypothese) durch ihre Einfachheit sehr überrascht». Selbst für diejenigen, die ihr ablehnend gegenüberstehen, müsse sie Interesse haben, «weil die gemeinsamen Folgerungen jeder mechanischen Wärmetheorie bei ihr so leicht und schlagend herausspringen ${ }^{72}$ Noch die heutigen elementaren Darstellungen der kinetischen Gastheorie lassen ihren Ursprung in KRöNIGS Abhandlung aus dem Jahre 1856 erkennen. Nachdem diese sich hundert Jahre lang als Grundlage der neuzeitlichen Wärmelehre bewährt hat, mag es billig erscheinen, die Frage nach den Lebensdaten des Autors zu stellen.

\section{Biographische Notizen zu August Karl Krönig}

Es war August KarL KrönIg versagt, weiter an Entwicklung und Ausbau der kinetischen Wärmetheorie teilzunehmen. Ein schweres Leiden zwang ihn zum Verzicht. Schon zu Lebzeiten geriet er in Vergessenheit. Daher fließen die biographischen Quellen spärlich - der an die Dissertation angehängte Lebenslauf, einige eingestreute Textstellen in seinen Schriften, die kurzen Angaben in Poggendorffs biographisch-literarischem Handwörterbuch $^{73}$. Nach diesen größtenteils eigenen Mitteilungen ist Krönig am 20. September 1822 in Schildesche, einem westfälischen Städtchen in der Nähe Bielefelds, als Sohn eines Pfarrers und als das sechste von sieben Kindern geboren. Er besucht das Gymnasium in Bielefeld und bezieht 1839 nach dem Abitur die Universität Bonn. Zunächst sucht er nach einer ihm gemäßen

72 s. Fortschritte der Physik XII (1859) 352.

73 Band I (1863) Sp. 1320 f.; Band III (1898) Sp. 752. 
Richtung, beschäftigt sich mit klassischer Philologie, mit Geschichte und Kulturgeschichte, mit Sanskrit und indischer Literatur und mit altorientalischen Kulturen. Gleichzeitig hört er Vorlesungen über Philosophie, Chemie, Mineralogie und Mathematik. Erst als er im vierten Semester nach Berlin geht, erfolgt die Konzentration auf die naturwissenschaftlichen und die mathematischen Fächer, die hier durch hervorragende Dozenten vertreten werden. Krönig nennt Heinr. Rose, Mitscherlich und Rammelsberg, Heinr. Dove, Gustav Magnus und Dirichlet unter seinen Lehrern. 1845 promoviert er mit einer chemischen Arbeit ${ }^{74}$.

Von nachhaltigem Einfluß wird die Teilnahme an den berühmten physikalischen Kolloquien, in welchen Gustav Magnus die damalige junge Berliner Physikergeneration um sich schart. Aus diesem erlesenen Kreise geht 1845 die «Physikalische Gesellschaft zu Berlin» hervor. Die jungen Gelehrten machen es sich zur Aufgabe, die schier unüberschaubare Fülle an physikalischem Schrifttum dem Fachmann in einem Referatenblatt zugänglich zu machen. Es wird eine Zeitschrift herausgegeben, die in Kurzreferaten über die in der Literatur auftretenden physikalischen Arbeiten berichtet und die Fortschritte der Physik genannt wird. Dem Vorbericht zum ersten Bande, in welchem die Gesellschaft Professor Gustav Magnus als dem «Urheber der Idee » ihren Dank ausspricht, schließt sich eine Liste der 53 Mitglieder an. Sie enthält bekannte Namen, darunter: Dr. W.Beetz, später Professor der Physik in München, Emil du Bors-Reymond, E. W. Brücke, der 1848 als Professor der Physiologie nach Königsberg, 1849 nach Wien geht, Helmholtz, Werner Siemens, damals noch Artillerieleutnant, Mechanikus Halske, Siemens' Mitarbeiter und Mitbegründer der bekannten Firma, G.H. Wiedemann und auch Dr.A. Krönig. Fast alle der Obengenannten haben 1845 das dreißigste Lebensjahr noch nicht erreicht. 1848 ist Krönig Schriftführer der Gesellschaft. Seine Neigung zu literarischer Tätigkeit ist groß. Die ausländischen Veröffentlichungen erscheinen noch unzugänglicher als die deutschen. Um abzuhelfen, gibt er ein dreibändiges Werk Journal für Physik und physikalische Chemie des Auslandes heraus, das 1851 erscheint und wichtige fremdsprachige Arbeiten der neueren Zeit in Übersetzung bringt. Seit 1855 redigiert er die Fortschritte der Physik, zunächst in Gemeinschaft mit BEEтz, später, als dieser einer Berufung nach auswärts folgt, als alleiniger Redakteur. 1859 muß er die Redaktion aus gesundheitlichen Gründen aufgeben.

${ }^{74}$ Diss. de acidi chromici salibus crystallinis, Berol. 1845. 
Neben der literarischen Beschäftigung ist Krönig auch als Lehrer der Mathematik und Naturwissenschaften tätig, 1850 als Hilfslehrer am Kölnischen Realgymnasium zu Berlin, 1852 als Lehrer an der königlichen Realschule, einer mit dem Friedrich-Wilhelms-Gymnasium verbundenen Lehranstalt. Er wird dort Oberlehrer. Später erhält er den Professortitel. 1861 kann er auch den Schuldienst nicht mehr versehen. Ein Nervenleiden, das seine Augen und seine Füße erfaßt, macht das unmöglich ${ }^{75}$. Es handelt sich offensichtlich um Anzeichen von multipler Sklerose.

Der Abschied von der Schule mag Krönig nicht schwer gefallen sein; denn seine Fähigkeiten als Lehrer schätzt er nicht hoch ein. Er spricht von seiner «Besessenheit», die Dinge dem Schüler in einer ganz bestimmten Form einzuprägen, die er nun einmal für die geeignete hält. Damit quält er sich und die Schüler ${ }^{76}$.

Zu Beginn seiner Krankheitszeit schreibt Krönig Monographien. Ein Lehrbuch Die Chemie, bearbeitet als Bildungsmittel für den Verstand (Berlin 1864) findet keine gute Aufnahme. Der Hang des Verfassers zur Vereinfachung, Schematisierung, zu einer endgültigen Form wird als beengend für den Unterrichtenden empfunden. Der nötigen Bewegungsfreiheit in der Darstellungsweise sei kein Spielraum eingeräumt. Und auch der Schüler werde durch dieses Lehrbuch nicht zu selbständiger gedanklicher und experimenteller Arbeit angeleitet. Überdies täusche das Streben des Verfassers, die Beobachtungsergebnisse durch Abrundung in präzise Gesetze zu fassen - in der Gastheorie so erfolgreich angewandt, aber nicht überall in der Chemie glücklich zu handhaben -, über wahrhafte Schwierigkeiten hinweg und gebe Anlaß zu unerlaubten Vorurteilen. Es entstehe ein falsches Bild von der Chemie, als sei diese ganz im Fluß begriffene Wissenschaft bereits abgeschlossen und ohne ungelöste Probleme. Vor allem treffen willkürliche Veränderungen der Begriffsinhalte für eingeführte Ausdrücke, z.B. für Atom und Molekül, auf Widerstand. Kritik und Antikritik führen zu heftiger Polemik ${ }^{77}$.

Als letztes Werk in Buchform erscheint eine religionsphilosophische Schrift Über das Dasein Gottes und das Glück des Menschen, materialistischerfahrungsphilosophische Studien ..., Berlin 1874. Der Krankheitszustand,

\footnotetext{
${ }^{75}$ s. A. KRöNIG, Wie kritisirt man chemische Lehrbücher? 1865, S. 36.

76 l.c., S. 37.

77 s. u. a. Siewert in Z. ges. Naturwiss. 23 (1864) 254; Lexis in Z. Chem. Pharmacie 7 (1864) 222; A.K. KRöNIG, Wie kritisirt man chemische Lehrbücher? Berlin 1865.
} 
inzwischen zur vollständigen Lähmung fortgeschritten, läßt eine zusammenhängende Fassung nicht mehr zustande kommen. Das Buch enthält in aphoristischer Form Betrachtungen und Lebensweisheitsregeln. Die meisten Abschnitte sind in den Jahren der Krankheit datiert. Die starrsinnige Verteidigung des eigenen Standpunktes, die Intoleranz gegenüber der Meinung anderer, die Neigung zu Polemik bestimmen auch hier den Gesamteindruck. Den Kernpunkt bildet, wie schon aus dem Titel hervorgeht, die Diskussion über die Existenz Gottes. Die Betrachtung der Natur, zumal der organischen, zwingt Krönig zu teleologischer Weltanschauung, zum Glauben an einen persönlichen Gott, an ein denkendes Wesen, ausgerüstet mit einer im Vergleich zur menschlichen unvergleichbar hohen Intelligenz. Andererseits kann es sich offenbar nicht um ein vollkommenes Wesen, etwa im Sinne der christlichen Religionen, handeln. In der Welt müßte es anders zugehen, wenn sie das Werk eines allmächtigen, allwissenden, allgütigen Gottes wäre. So wird ein Kompromiß geschlossen, und der Verfasser stellt als einzige ihm mit dem gesunden Menschenverstand vereinbar erscheinende Lösung die farblose Erscheinung eines Weltkonstrukteurs hin, der am Weltgeschehen im übrigen unbeteiligt ist. Es liegt auf der Hand, daß Krönigs Idee von der Gottheit wenig geeignet war, zahlreiche Jünger zu werben. Ohne die Größe des göttlichen Demiurgen, die Erhabenheit des weltenlenkenden Prinzips war sie denen, die im Glauben Gott suchen, nicht Hüter des Mysteriums vom Leben und vom Tode, nicht Zuflucht und Stütze in den Nöten des Daseins. Die Materialisten aber, denen diese dürre Gestalt noch unannehmbarer erscheinen mußte als der Gott der Offenbarungsreligionen, konnten ihrer ganz entbehren. So ist es bemerkenswert, daß das Buch gerade vom religiösen Standpunkt aus eine relativ milde Priesse gefunden hat. Man dankt es dem Autor, daß er, der in den Naturwissenschaften Bewanderte, sich entgegen BüchNer ${ }^{78}$ und anderen für die Existenz eines persönlichen Gottes einsetzt, wenn dieser auch nicht in allen Hinsichten annehmbar sei. Nicht ohne Bewegung vermerkt man die Bemühungen des schwer Leidenden, den Mitmenschen eine aus eigenster Erfahrung erwachsene Beglückungslehre zu vermitteln. Man erkennt den tapferen Streiter an, der trotz aller Widerwärtigkeiten das Leben bejaht und seine geistige Kraft und Unabhängigkeit bewahrt hat.

78 Ludwig Büchner (1824-1899), Hauptvertreter des Materialismus, Verfasser zahlreicher Schriften. Sein bekanntestes Werk, Kraft und Stoff, 1855, 21. Auflage 1904, wurde in fünfzehn Sprachen übersetzt. 